\title{
A young lady with MELAS syndrome: a sporadic case
}

\author{
KAjantha ${ }^{1}$, MSG De Silva ${ }^{2}$ \\ ${ }^{1}$ Registrar in Medicine, ${ }^{2}$ Consultant Physician, National Hospital of Sri Lanka, Colombo
}

\section{Introduction}

Mitochondrial myopathy Encephalomyopathy Lactic Acidosis and Stroke-like syndrome (MELAS) is a rare neurodegenerative disease, characterized by recurrent stroke-like episodes, lactic acidosis, bilateral occipitoparietal infarction and basal ganglion calcification. Only a few cases have been documented worldwide.

\section{Case report}

A 30 year-old lady presented with acute severe headache, tinnitus, blurred vision and weakness followed by two episodes of generalized tonic clonic fits. Symptoms worsened over a period of 48 hours. Her first presentation with a similar illness was two years ago and since then she has hearing impairment.

On examination, she was unconscious with grade III weakness in all four limbs, exaggerated deep tendon reflexes and right extensor plantar response.

Her random blood sugar, full blood count, arterial blood gas analysis and cerebrospinal fluid analysis were normal. Urine full report showed proteinuria $(++)$ and no cells. Blood culture and urine culture were negative. ESR was $110 \mathrm{~mm}$ for the first hour but ANA and thrombophilic screening including lupus anticoagulant, anticardiolipin, antibodies IgG and IgM were negative. 
CT scan of the brain showed bilateral basal ganglion calcification (Figure 1) and diffuse cerebral infarction involving occipito-parietal regions (Figure 2). The ECG showed WolffParkinson-White Syndrome (WPW) type B but chest X-Ray and echocardiography were normal. Tests for HIV were negative.

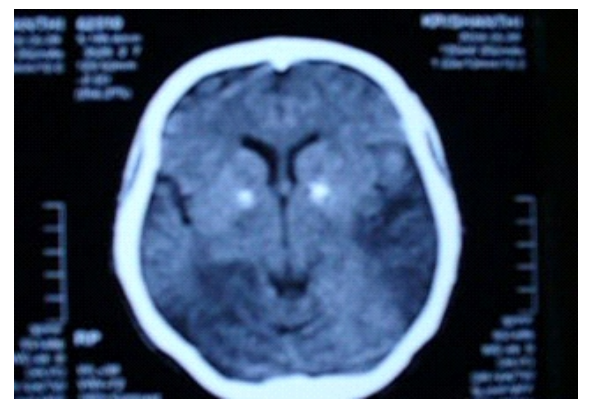

Figure 1 - CT scan of the brain showing calcification of basal ganglia

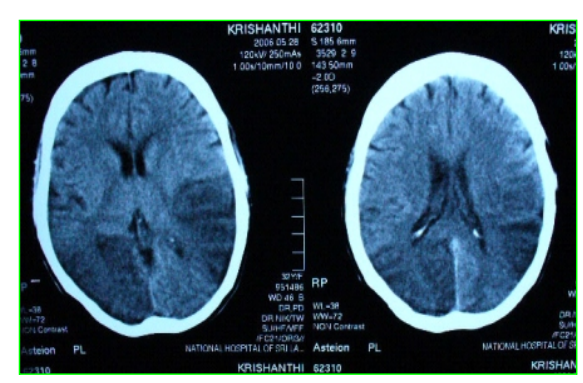

Figure 2 - CT scan of the brain showing diffuse cerebral infarction involving occipitoparietal regions

A tentative diagnosis of meningoencephalitis was made and she was commenced on Aciclovir and IV ceftriaxone but there was no improvement. Serum lactic acid concentration was $110.4 \mathrm{mg} / \mathrm{dL}$ (Normal 4.5-19.8). CPK was normal. Muscle biopsy showed dysmorphic muscle fibers under the light microscope. Audiometry showed neurosensory deafness. She achieved a delayed partial recovery over a period of three months, but was dementic and mute most of the time. She was discharged on niacin and vitamin $\mathrm{B}_{1}$.

\section{Discussion}

MELAS is a rare progressive multisystem disease, presenting either sporadically or among members of affected maternal pedigree. In USA no estimates of MELAS mutation are available. However, it is 10.2 per 100,000 in adult Finnish population and 1 per 13000 in Northern England. It has high morbidity and mortality. No sex predilection exists. About $80 \%$ of patients have a heteroplasmic $\mathrm{A}$ to $\mathrm{G}$ point mutation in Dihydrouridine molecules and it is detected in mitochondrial DNA [1,2]. Metabolic stroke-like episodes may be vascular or parenchymal due to a transient dysfunction of oxidative phosphorylation and consequently increased free radicals, vasoconstriction and decreased oxygen availability [3].

The myopathy manifests with weakness and easy fatigability. Encephalopathy may progress to dementia and apathy. The strokelike episodes are the hallmark of the disease -associated initially with vomiting, headache and seizures and later with hemiplegia. The visual complaints are due to either ophthalmoplegia or blindness which may occur as a result of optic atrophy, lesions in the area of the brain concerned or retinal pigmentation. Diabetes mellitus is a common manifestation [4]. The dilated or hypertrophic cardiomyopathy and WPW may be present in some patients. Peripheral neuropathy, neurosensory deafness, gait ataxia, psychiatric disorder and neuroradiological features such as cerebral infarction, cerebral atrophy and calcification of basal ganglia have also been reported in some cases [3,5].

The clinical diagnosis of MELAS is based on the presence of the following 3 major features;

1. Stroke-like episodes, typically before 40 years of age.

2. Encephalopathy with seizures or dementia.

3. Mitochondrial myopathy, evidenced by lactic acidosis or ragged red fibers and the presence of two of the following features;

- Normal early psychomotor development.

- Recurrentheadaches.

- Recurrent vomiting [5]. 


\section{Case Reports}

The muscle biopsy under H\&E stain shows the changes due to myopathy. Ragged red fibers are the hallmark of MELAS, but it needs a special stain - Brilliant Red. The electron microscopy shows increase in number and size of mitochondria $[3,5]$.

Management is conservative. The vitamin supplements like Co enzyme Q10, Vit $\mathrm{K}_{3}$ and $\mathrm{K}_{4}$, riboflavin and niacin have been suggested to support respiratory chain enzymes in mitochondria, but no therapy is of proven efficacy. The genetic screening and counselling should be done at least in the first degree relatives. $[3,5,6]$

This case highlights the need to be aware of uncommon conditions that can have a common clinical presentation.

\section{References}

1. Borner GV, Zeviani M, Tiranti V, et al. Decreased aminoacylation of mutant tRNAs in MELAS but not in MERRF patients. Hum Mol Genet 2006; 9(4): 467-75.

2. Tanahashi C, Nakayama A, Yoshida M, et al. MELAS with the mitochondrial DNA 3243 point mutation: a neuropath logical study. Acta Neuropathol (Berl) 2000 Jan; 99(1): 31-8.

3. Scaglia F, et al MELAS syndrome; eMedicine updated 2006; October 25.

4. Joko $\mathrm{T}$, Iwashige $\mathrm{K}$, Hashimoto $\mathrm{T}$, et al. A case of mitocho ndrial encephalomyopathy, lactic acidosis and stroke-like episodes associated with diabetes mellitus and hypothalamo pituitary dysfunction. Endocrinology Journal 1997; Dec 44(6): 805-900 and Mar 1;9(4): 467-75.

5. Dimauro S, Irano M. MELAS Gene reviews, update 13 October 2005

6. Scaglia F, Northrop JL. MELAS, A review of treatment options CNS drugs 2006; 20(6): 443-64. 